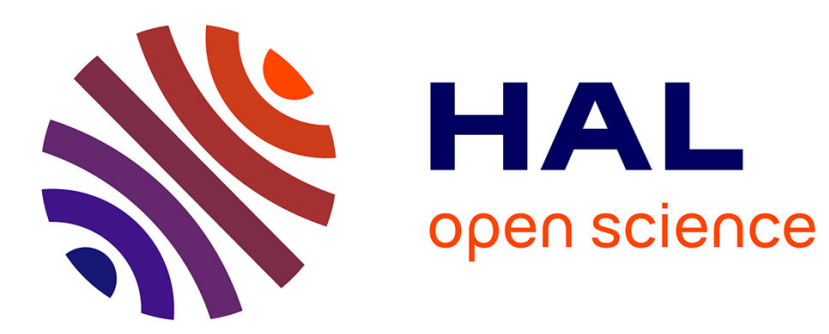

\title{
Quantum-like model of brain's functioning: decision making from decoherence
}

Masanari Asano, Masanori Ohya, Yoshiharu Tanaka, Irina Basieva, Andrei Khrennikov

\section{- To cite this version:}

Masanari Asano, Masanori Ohya, Yoshiharu Tanaka, Irina Basieva, Andrei Khrennikov. Quantumlike model of brain's functioning: decision making from decoherence. Journal of Theoretical Biology, 2011, 281 (1), pp.56. 10.1016/j.jtbi.2011.04.022 . hal-00708524

HAL Id: hal-00708524

https://hal.science/hal-00708524

Submitted on 15 Jun 2012

HAL is a multi-disciplinary open access archive for the deposit and dissemination of scientific research documents, whether they are published or not. The documents may come from teaching and research institutions in France or abroad, or from public or private research centers.
L'archive ouverte pluridisciplinaire HAL, est destinée au dépôt et à la diffusion de documents scientifiques de niveau recherche, publiés ou non, émanant des établissements d'enseignement et de recherche français ou étrangers, des laboratoires publics ou privés. 


\section{Author's Accepted Manuscript}

Quantum-like model of brain's functioning: decision making from decoherence

Masanari Asano, Masanori Ohya, Yoshiharu Tanaka, Irina Basieva, Andrei Khrennikov

PII:

S0022-5193(11)00222-0

DOI: doi:10.1016/j.jtbi.2011.04.022

Reference: YJTBI 6453

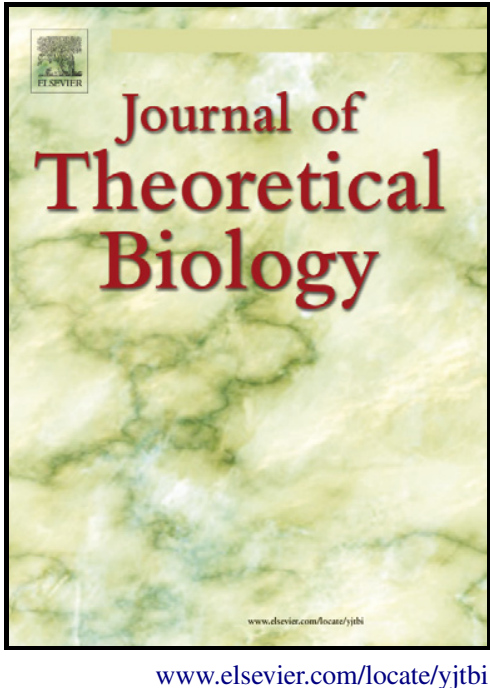

To appear in: $\quad$ Journal of Theoretical Biology

Received date: 2 December 2010

Revised date: 19 February 2011

Accepted date: 22 April 2011

Cite this article as: Masanari Asano, Masanori Ohya, Yoshiharu Tanaka, Irina Basieva and Andrei Khrennikov, Quantum-like model of brain's functioning: decision making from decoherence, Journal of Theoretical Biology, doi:10.1016/j.jtbi.2011.04.022

This is a PDF file of an unedited manuscript that has been accepted for publication. As a service to our customers we are providing this early version of the manuscript. The manuscript will undergo copyediting, typesetting, and review of the resulting galley proof before it is published in its final citable form. Please note that during the production process errors may be discovered which could affect the content, and all legal disclaimers that apply to the journal pertain. 


\title{
Quantum-like model of brain's functioning: decision making from decoherence
}

\author{
Masanari Asano, Masanori Ohya and Yoshiharu Tanaka \\ Department of Information Sciences, Tokyo University of Science \\ Yamasaki 2641, Noda-shi, Chiba, 278-8510 Japan \\ Irina Basieva and Andrei Khrennikov \\ International Center for Mathematical Modelling \\ in Physics and Cognitive Sciences \\ Linnaeus University, Växjö, S-35195 Sweden \\ Email: Andrei.Khrennikov@lnu.se
}

\begin{abstract}
We present a quantum-like model of decision making in games of the Prisoner's Dilemma type. By this model the brain processes information by using representation of mental states in a complex Hilbert space. Driven by the master equation the mental state of a player, say Alice, approaches an equilibrium point in the space of density matrices (representing mental states). This equilibrium state determines Alice's mixed (i.e., probabilistic) strategy. We use a master equation which in quantum physics describes the process of decoherence as the result of interaction with environment. Thus our model is a model of thinking through decoherence of the initially pure mental state. Decoherence is induced by the interaction with memory and the external mental environment. We study (numerically) the dynamics of quantum entropy of Alice's mental state in the process of decision making. We also consider classical entropy corresponding to Alice's choices. We introduce a measure of Alice's diffidence as the difference between classical and quantum entropies of Alice's mental state. We see that (at least in our model example) diffidence decreases (approaching zero) in the process of decision making. Finally, we discuss the problem of neuronal realization of quantum-like dynamics of in the brain; especially roles played by lateral prefrontal cortex or and orbitofrontal cortex.
\end{abstract}

Keywords: Decision making, dynamics of mental state, quantum-like model, interaction with memory, decoherence, quantum master equation, neuronal paradigm

\section{Introduction}

The idea that the mathematical formalism of quantum information theory can be used to describe information processes in the brain was elaborated in a series of papers, see [1]-[14]. This approach is based on the fundamental conjecture that the real physical brain developed an ability to represent the probabilistic information in complex linear space, by complex vectors (pure quantum-like mental states) or more generally density operators (mixtures of pure quantum-like mental states. ${ }^{1}$ (This paper contains a brief appendix on mathematics of quantum mechanics which may be useful for biologists, see also book [11].)

\footnotetext{
${ }^{1}$ In section 5 we discuss the problem of neuronal realization of quantum-like dynamics of in the brain; especially roles played by lateral prefrontal cortex or and orbitofrontal cortex. We use studies on neuronal correlates observed in the process of decision making [15],

We know well that quantum information processing can be simulated on the classical computer. Therefore our model need not be based on physically quantum brain as, e.g., the models of Homeroff [19], [20] and Penrose [21], [22]. Since the brain has huge computa-

[16]. Of course, these are very preliminary considerations on a possible neuronal realization of the quantum-like dynamics of decision making. However, it is useful to initiate such a discussion as soon as possible to attract attention of neuroscientists and psychologists to the problem of neuronal realization of the quantum-like dynamics of decision making. We also mention that quantum-like probabilistic effects can be found not only in functioning of advanced cognitive systems, but even in cell's biology. For example, we can point to the well known glucose effect on $E$. coli growth; destructive interference of two factors: the presence of lactose and glucose in a E. coli cell [17]. It is also well known that a dental epitherial cell grows in a medium as it is (no differentiation). A dental mesenchymal cell grows similarly. However, this two contexts are incompatible, see [18] for statistical data. Their unification induces quantum-like interference. 
tional resources (a "neural computer" with 300 billions 71 of processing units), the main reason for usage of the 72 quantum representation of information may be not in- 73 creasing the speed of computations. The main distin- 74 guishing feature of the quantum information represen- 75 tation is a possibility to operate with superpositions of 76 states. We explore this feature of quantum information 77 processing. From the very beginning we want to dis- 78 tance from models of cognition based on quantum phys- 79 ical carriers of information. It seems that it is impossi- 80 ble to make these models consistent with the neuronal 81 paradigm of cognition. We like to combine quantum 82 information theory with the neuronal paradigm. A pos- 83 sible physical realization of quantum-like processing of 84 information by the brain operating with classical elec- 85 tromagnetic fields was presented in [23].

A few years ago J. Busemeyer et al. [2] noticed that ${ }_{87}$ quantum-like models of decision making can be used 88 to explain disjunction effect in experiments of cognitive 89 psychology. In particular, he reproduced statistical data 90 from experiments of the Prisoner's Dilemma (PD) type 91 obtained by Shafir and Tversky [24], [25], see also [26]. 92 Busemeyer's quantum-like model of decision making in $9_{3}$ PD is a psychological analog of the quantum model for 94 the hydrogen atom. Of course, both models are over- 95 simplified and to be applied to more complicated phe- 96 nomena, they should be essentially modified. However, 97 both express the main distinguishing features of corresponding theories (quantum physics and quantum-like cognitive psychology). Therefore in this paper we proceed with PD. Following to tradition of quantum infor- 10 mation theory we call players Alice and Bob, see section 2 for details.

Our aim is to describe the process of decision mak- 104 ing in the brain; in particular, interaction with mem- 105 ory. Quantum-like models of decision making were pre- 106 sented in a series of papers [1]-[3], [6], [8]-[14].

Dynamical models of decision making are of the main 108 interest for us. We recall that, in a few papers [1], [2], 109 [12], the process of decision making was described by 110 Schrödinger's evolution of the mental state. The lat- 111 ter was assumed to be a pure state (mathematically it is 112 represented by a normalized vector of complex Hilbert 113 space). In this paper we shall explore in more detail the ${ }_{114}$ idea [14] that decision making has to be represented by a 115 more complicated dynamics which describes the evolu- 116 tion of quantum (or more generally quantum-like state) 117 interacting with an environment. Such dynamics plays 118 an important role in quantum physics. Its fundamen- 119 tal feature is transformation of pure states (described by 120 complex vectors) into mixed states (described by den- 121 sity matrices) - decoherence. In our cognitive model 122 memory is an important part of the "mental environment" which induces decoherence of a pure state, Alice's state of mind before staring the process of decision making. In this paper we do not model the process of interaction with memory; as often in quantum information theory we represent memory (as well as the external mental environment) as a bath, in our case a "mental bath." In a future paper we plan to model this process in more detail by using the apparatus of quantum Markov chains, cf. [6].

In quantum physics interaction of a quantum system with a bath is described by a quantum version of the master equation. Quantum Markovian dynamics given by the Gorini-Kossakowski-Sudarshan-Lindblad (GKSL) equation, see e.g. [27] for detail, is the most popular approximation of quantum dynamics in the presence of interaction with a bath. We remind shortly the origin of the GKSL-dynamics. The starting point is that the state of a composite system, a quantum system $s$ combined with a bath, is a pure quantum state, complex vector $\Psi$, which evolution is described by Schrödinger's equation. This is an evolution in a Hilbert space of the huge dimension (since a bath has so many degrees of freedom). The existence of the Schrödinger dynamics in the huge Hilbert space has a merely theoretical value. Observers are interested in the dynamics of the state $\phi_{s}$ of the quantum system $s$. The next fundamental assumption in derivation of the GKSL-equation is the Markovness of the evolution, the absence of long term memory effects. It is assumed that interaction with the bath destroys such effects. Thus, the GKSL-evolution is Markovian evolution. Finally, we point to the condition of the factorizability of the initial state of a composite system (a quantum system coupled with a bath), $\Psi=\phi_{s} \otimes \phi_{\text {bath }}$, where $\otimes$ is the sign of the tensor product. Physically factorization is equivalent to the absence of correlations (at the beginning of evolution; later they are induced by the interaction term of Hamiltonian the generator of evolution). One of distinguishing features of the evolution under the mentioned assumptions is the existence of one or a few equilibrium points. The state of the quantum system $s$ stabilizes to one of such points in the process of evolution; a pure initial state, a complex vector $\psi_{s}$, is transformed into a mixed state, a density matrix $\rho_{s}(t)$. In contrast to the GKSL-evolution, the Schrödinger evolution does not induce stabilization; any solution different from an eigenvector of Hamiltonian will oscillate for ever. Another property of the Schrödinger dynamics is that it always transfers a pure state into a pure state, i.e., a vector into a vector. And we want to obtain mixed states, diagonalized in the basis corresponding to the decision operator. The GKSL- 
evolution gives such a possibility.

In the process of decision making the brain selects a 172 pure mental state describing possible decisions of the ${ }_{173}$ problem under consideration and drives this state - de- 174 note it by $\phi_{A}$ (superposition of possible decisions of 175 Alice in her game with Bob). The state of the mental 176 environment is represented by another complex vector, 177 say $\phi_{B}$. In general, this is a huge mental state represent- 178 ing all superpositions in memory and even permanent 179 supply of superpositions created by the brain through 180 its interaction with the environment. However, if Alice is concentrated on her strategy of play with Bob, we can restrict $\phi_{B}$ to Alice's mental image of the possible actions of Bob. In reality $\phi_{B}$ belongs to complex Hilbert space of a large dimension. Therefore the standard assumption used in the derivation of the GKSLequation is fulfilled. (In this paper, we consider a toy ${ }_{18}$ model in which $\phi_{B}$ is two dimensional, representing su- ${ }_{186}$ perposition of possible actions of Bob created in Alice's brain. Thus formally one of the most important assump- ${ }_{187}$ tions of derivation of the GKSL-equation is not fulfilled. However, more detailed analysis shows that, in fact, in quantum physics the dimension of a bath is not crucial. The crucial property of a bath is that it is very stable to fluctuations in the quantum system $s$ interacting with it. This assumption is fulfilled if Alice's image of possible actions of Bob is sufficiently stable with respect to fluctuations of the state of her possible actions.) The assumption of Markovness of the mental state evolution in decision making is natural. To proceed quickly to a decision, Alice must ignore the history of her reflections on possible actions with respect to Bob. ${ }^{2}$ An input from (long-term) memory or mental environment destroys (working) memory of her reflections. (Working memory does not preserve a long chain of Alice's reflections.) Finally, we can assume that the initial composite state is factorized, i.e., correlations between Alice's image of Bob and her possible actions are created in the 195 process of decision making. Under these assumptions 196 we can model the process of decision making by using ${ }_{197}$ the GKSL-equation.

The mental state representing possible actions of Al- 199 ice stabilizes to one of equilibrium points of the GKSL- 200 dynamics. (In the mathematical model stabilization is 201 achieved only in the limit $t \rightarrow \infty$. However, in real- 202 ity the brain cannot wait too long. We can assume the 203 presence (in the brain) of a threshold $\epsilon$ which is used 204 to terminate the process of stabilization of the mental 205

${ }^{2}$ Such reflections are processed in her working memory. So, we ${ }^{207}$ discuss Markovness of working memory. Of course, in our model 208 long-term memory is not ignored; it is a part of the mental bath. state to a point of equilibrium, see also Remark 3.) A model equation considered in this paper has a single equilibrium point. Thus Alice elaborates the unique solution (which depends only on the mental environment, in particular, memory). However, in general the GKSLequation can have a few different equilibrium points. In such a case depending on the initial state of mind Alice can obtain different solutions of the same problem. Such equations with a richer structure of equilibrium points will be studied in one of coming papers.

\section{Prisoner's Dilemma}

In the paper of [14], we designed a quantumlike model for decision-making process in two-player games. This section explains briefly how a player in our model decides his own action, cf. also Busemeyer et al. [2].

\subsection{Pay-off Table of Two-player Game}

Let us consider a two-player game with two strategies. We name two players Alice ("A") and Bob ("B"). Two strategies which $\mathrm{A}$ and $\mathrm{B}$ can choose are denoted by " 0 " and " 1 ". The following table shows payoffs assigned to possible four consequences of " $0_{A} 0_{B}$ ", " $0_{A} 1_{B}$ ", " $1_{A} 0_{B}$ " and " $1_{A} 1_{B}$ ". Here, $a, b, c$ and $d$ denote

\begin{tabular}{|c|c|c|}
\hline$A \backslash B$ & $0_{B}$ & $1_{B}$ \\
\hline $0_{A}$ & $(a \backslash a)$ & $(b \backslash c)$ \\
\hline $1_{A}$ & $(c \backslash b)$ & $(d \backslash d)$ \\
\hline
\end{tabular}

${ }^{193}$ the values of pay-offs.

For example, a game of prisoner's dilemma (PD) type is characterized by the relation of

$$
c>a>d>b .
$$

For the player A, his pay-off will be $a$ or $c$ if the player B chose " 0 " and $b$ or $d$ if the player B chose " 1 ". In the both cases, from the relations of $c>a$ and $d>$ $b$, he can obtain larger pay-offs if he choose 1 . The situation is same for the player B. Conventional game theory concludes that in PD game a "rational" player, who wants to maximize his own payoff, always chooses " 1 ".

However, the above discussion does not explain completely the process of decision-making in real player's mind. Actually, as seen in statistical data in some experiments on so called disjunction effect [24]-[26] real players frequently behave "irrationally". Our model is an attempt to describe such real player's behaviors by some mathematical formalism. Our model is a 

paper "quantum-like model" which is derived from basic con- 259 cepts of quantum mechanics, but not an expansion of 260 conventional game theory.

Remark 1. (Rationality) The conventional decision ${ }_{262}$ making theory is based on the notion of rationality ${ }_{263}$ which is closely related to the Savage sure thing prin- ${ }^{264}$ ciple (SSP) [29] (one of the basic principles of mod- 265 ern economics) having a large impact to cognitive psy- 266 chology and cognitive science, see [12], [11] for popu- 267 lar presentations for biologists and psychologists. This 268 principle matches well our intution. This is a gen- 269 eral principle of consistency of our decisions. If, e.g., 270 Noboru plans to buy some shares under the condition 271 that an event $A$ happens and at the same time he plans 272 to buy these shares even under the condition that A does 273 not happen, then it seems natural for him to buy them 274 even if there is no information whether $A$ happened or 275 not. This principle of consistency of decision making 276 is related to the very foundation of classical probabil- 277 ity theory, the formula of total probability, see [2], [12], 278 [11], [9] for details. The formula of total probability 279 is the basis of the Bayesian approach to decision mak- 280 ing. Therefore violation of SPP (which is equivalent ${ }_{281}$ to violation of the formula of total probability) implies 282 impossibility to use the Bayesian scheme of decision ${ }_{283}$ making. In game theory the approach developed by von ${ }_{284}$ Neumann and Morgenstern [30] is also closely related 285 to SSP. In particular, the use of the mini-max principle 286 and the Nasch equilibrium are characteristic for "ratio- 287 nal players", rational in the sense of SSP. We remark 288 that SSP and the von Neumann-Morgenstern [30] ap- 289 proach to game theory had been criticized from the first 290 days of their creation. Many bright thinkers pointed 291 to inadequacy of SSP-rationality to real brain's func- 292 tioning. ${ }^{3}$ However, a better mathematical model had 293 not been created. In particular, we can point to a re- 294 cent detailed analysis of SSP-rationality [31]. The au- 295 thors of this paper rightly couple SSP-rationality with 296 the Bayesian approach to decision making. They criti- 297 cized the Bayesian scheme of decision making pointing 298 to numerous experiments in cognitive science and psy- 299 chology demonstrating inapplicability of this scheme. 300 However, the conclusion of the authors of [31] - it is im- 301 possible to create a general formal mathematical model 302 of decision making - is not acceptable for us. We gen- 303 eralize the conventional Bayesian scheme of decision 304 making by operating with a priori probabilistic amplitudes, instead of Bayesian a priori probabilities. Thus, opposite to [31], we claim that, in spite of violations of

${ }^{3}$ Thus the critique of SSP-rationality is not the main aim of our
SSP, the notion of rationality can be formalized.

Remark 2. (Quantum games) Theory of quantum games is an important part of quantum information theory, see, e.g., [32], [33]. In such a game theory quantum carriers of information, e.g., photons, are used as a source of randomness. Quantum game theory is typically coupled to quantum physics. In contrast, we consider games played by "classical" macroscopic players. We do not assume that any really quantum source of randomness is involved in a game. ${ }^{4}$ Therefore, opposite to the traditional quantum game theory, we do not assume that Alice and Bob e.g. share entangled photons. This is the basic interpretational difference between "physical quantum game theory" and our quantum-like game theory. This difference in interpretations plays a crucial role in application of quantum mathematics to real games. Opposite to e.g. [33], we consider the real PD and not a quantum PD-game which is based on sharing of entangled quantum systems by "prisoners." (Experts in "physical quantum game theory" typically stress its role in future world of quantum technologies.) Our quantum-like game approach has violation of the formula of total probability as the point of departure. So, we do not speculate what kind of gambling would be popular after invention of quantum computers and communication. Finally, we point to an interesting publication of Cheon and Tsutsui [34] which may serve as a bridge between the traditional theory of quantum games and our quantum-like game theory. They rightly stressed the role of non-factorizability of probabilities in generation of nonclassical strategies. The condition of non-factorizability can be formulated without the usage of the formalism of quantum mechanics. There is no need to couple this condition to entanglement of states of quantum carriers of information. Therefore the Cheon-Tsutsui approach can be considered as quantumlike. We now turn to the mathematical structures of quantum and quantum-like game theories. If one forget about interpretation differences ${ }^{5}$, then quantum and quantum-like games are based on the same mathematics - the mathematical formalism of quantum mechanics. The main problem of the "ordinary quantum game theory" is that it considers physical quantum systems as sources of randomness. Therefore all actions on these systems which are permitted by quantum mechanics can be used in a quantum game. All possible unitary trans-

\footnotetext{
${ }^{4}$ Although we do not reject completely physical quantum models of brain's functioning, e.g., based on entanglement in brain microtubules, we proceed without coupling to such models.

${ }^{5}$ I guess that for adherents of the conventional interpretation of quantum mechanics it would be impossible.
} 
formations are in usage as game strategies. Roughly 348 speaking in this framework it is possible to obtain everything. In contrast, quantum-like games under consideration are coupled to brain's functioning (at least to psychological functions). This stimulate dynamical approach to decision making, i.e., selection of strategies through quantum-like mental dynamics, see Busemeyer et al. [2], [12], Khrennikov [1], [11]. In [14] we made a new step: in the process of decision making the final state is determined not by a unitary evolution, but by a decoherence process described by the quantum master equation. This is an important extension of the mathematical formalism of quantum game theory. One of the ${ }_{349}$ main distinguishing features of the "quantum(-like) de- 350 coherence game theory" is non-invertibility of actions ${ }_{351}$ of players. Different initial states can produce the same ${ }_{352}$ output. This feature of our model matches well infor- ${ }_{353}$ mation processing by (macroscopic) biological systems. ${ }_{354}$ (Unitarity is not present in the macroworld. Therefore ${ }_{355}$ any model based on application of the quantum formalism to macrosystems has to contain a mechanism of violation of linearity; decoherence is the most natural one.)

\subsection{Complex Hilbert Space Representation of Player's Mind}

We present a model for the decision-making process in two-players games. ${ }^{6}$ We focus on player A's mind. In principle, the player $\mathrm{A}$ is not informed of which action the player B chooses. The player A will be conscious of two potentials of B's action simultaneously, and then he can not deny either of these potentials. In our model, this indeterminacy the player A holds is described by the following quantum superposition

$$
\left|\phi_{B}\right\rangle=\alpha\left|0_{B}\right\rangle+\beta\left|1_{B}\right\rangle \in \mathbf{C}^{2} .
$$

The values of $\alpha$ and $\beta$ are related to degrees of consciousness to B's actions (so to say, these are complex probabilistic amplitudes of A's intentions that B may make decisions 0 or 1 , respectively). We call this complex vector $\left|\phi_{B}\right\rangle$ a prediction state vector. (In accordance with the formalism of quantum mechanics, we assume $|\alpha|^{2}+|\beta|^{2}=1$.)

The player A who is getting to choose the action " 0 " will be conscious of two consequences of " $0_{A} 0_{B}$ " and " $0_{A} 1_{B}$ " with probability amplitudes of $\alpha$ and $\beta$. This situation is described with a vector from $\mathbf{C}^{2} \otimes \mathbf{C}^{2}$ given

\footnotetext{
${ }^{6}$ At the first stage it repeats the model of Busemeyer at al [2]. However, we restrict consideration to factorized initial state. This factorization will provide a possibility to apply decoherence dynamics.
}

by

$$
\begin{aligned}
\left|\Phi_{0_{A}}\right\rangle & =\alpha\left|0_{A} 0_{B}\right\rangle+\beta\left|0_{A} 1_{B}\right\rangle \\
& =\left|0_{A}\right\rangle \otimes\left|\phi_{B}\right\rangle
\end{aligned}
$$

Similarly,

$$
\left|\Phi_{1_{A}}\right\rangle=\left|1_{A}\right\rangle \otimes\left|\phi_{B}\right\rangle
$$

is given for the situation such that $\mathrm{A}$ is getting to choose "1". By using these state vectors $\left|\Phi_{0_{A}}\right\rangle$ and $\left|\Phi_{1_{A}}\right\rangle$, we define the following vector:

$$
\left|\Psi_{A}\right\rangle=x\left|\Phi_{0_{A}}\right\rangle+y\left|\Phi_{1_{A}}\right\rangle \in \mathbf{C}^{2} \otimes \mathbf{C}^{2},
$$

$\left(|x|^{2}+|y|^{2}=1\right)$. This state vector describes A's intensions to act. We call it a mental state vector (of A's intensions to act).

The player A's brain in this mental state chooses his own action probabilistically. His decision is described as "quantum measurements" of projectors corresponding to the vectors $\left|\Phi_{0_{A}}\right\rangle$ or $\left|\Phi_{1_{A}}\right\rangle$ on the state $\left|\Psi_{A}\right\rangle$. (Probabilities of " 0 " and " 1 " are given by $P\left(0_{A}\right)=P_{0_{A}}=|x|^{2}$ and $P\left(1_{A}\right)=P_{1_{A}}=|y|^{2}$.)

In our model, the decision-making process is described as a dynamics changing $|x|^{2}$ and $|y|^{2}$, and its dynamics has an equilibrium solution. Such stabilization of the mental state explains the following psychological activity in the player's mind: The player has two psychological tendencies, the one to choose 0 and the one to choose 1. Degrees of these two opposite tendencies change in his mind, and they become stable with balancing. (Fluctuations die and the definite probabilistic picture of the situation is created in A's mind).

The most simple dynamics of the stabilization of probabilities, the equations like chemical equilibration can be used in cognitive modelling:

$$
\begin{aligned}
& \frac{d}{d t} P_{0_{A}}=-k P_{0_{A}}+\tilde{k} P_{1_{A}}, \\
& \frac{d}{d t} P_{1_{A}}=k P_{0_{A}}-\tilde{k} P_{1_{A}} .
\end{aligned}
$$

The parameter $k(\tilde{k})$ corresponds to the velocity of the reaction from $0_{A}$ to $1_{A}$ (from $1_{A}$ to $0_{A}$ ), and in the equilibrium state, the probabilities $P_{0_{A}}$ and $P_{1_{A}}$ are given as

$$
P_{0_{A}}^{E}=\frac{\tilde{k}}{k+\tilde{k}}, P_{1_{A}}^{E}=\frac{k}{k+\tilde{k}} .
$$

The differential equations (5) are not described as a quantum dynamics. In fact, they give only a part of the complete system of quantum dynamical equations, the dynamics of the diagonal terms of the density matrix. We shall complete the system (5) and obtain a quantum dynamics; a system of differential equation for elements of the density matrix of the mental state, (12). 
2.3. Amplitudes of Velocities of Random Transitions in 413 Alice's Mind

As we have seen from Eq. (5), player's tendency to choose 1 or 0 is proportional to the velocity $k$ or $\tilde{k}$, and these parameters determine the stability solution (6). The choice of $k$ and $\tilde{k}$ is a very important issue in our model. We assume, their values are determined through comparison of possible consequences, $0_{A} 0_{B}, 0_{A} 1_{B}, 1_{A} 0_{B}$ and $1_{A} 1_{B}$. The player in our model will consider the following four kinds of comparisons:

$$
\begin{aligned}
0_{A} 0_{B} \underset{\tilde{k}_{1}}{\stackrel{k_{1}}{\rightleftharpoons}} 1_{A} 0_{B}, 0_{A} 1_{B} \underset{\tilde{k}_{2}}{\stackrel{k_{2}}{\rightleftharpoons}} 1_{A} 1_{B}, \\
0_{A} 1_{B} \underset{\tilde{k}_{3}}{\stackrel{k_{3}}{\rightleftharpoons}} 1_{A} 0_{B}, 0_{A} 0_{B} \underset{\tilde{k}_{4}}{\stackrel{k_{4}}{\rightleftharpoons}} 1_{A} 1_{B} .
\end{aligned}
$$

These comparisons are represented like the conditions of chemical equilibrium, each of which is specified by the reaction velocities, $k_{i}$ and $\tilde{k}_{i}$.

The velocities $k$ and $\tilde{k}$ should have the forms reflect- ${ }^{422}$ ing effects of the four comparisons and interferences be- ${ }^{428}$ tween them. In order to define these velocities in the ${ }_{429}$ appropriative forms, we introduce complex numbers $\mu \quad 430$ and $\tilde{\mu}$, which determine $k$ and $\tilde{k}$ by

$$
k=|\mu|^{2}, \tilde{k}=|\tilde{\mu}|^{2},
$$

and define these $\mu$ and $\tilde{\mu}$ (see [14] for details) as

$$
\begin{aligned}
& \mu \equiv|\alpha|^{2} \mu_{1}+|\beta|^{2} \mu_{2}+\alpha \beta^{*} \mu_{3}+\alpha^{*} \beta \mu_{4} \\
& \tilde{\mu} \equiv|\alpha|^{2} \tilde{\mu}_{1}+|\beta|^{2} \tilde{\mu}_{2}+\alpha^{*} \beta \tilde{\mu}_{3}+\alpha \beta^{*} \tilde{\mu}_{4} .
\end{aligned}
$$

Here $\mu_{i=1,2,3,4}$ and $\tilde{\mu}_{i=1,2,3,4}$ are complex numbers satisfying $\left|\mu_{i}\right|^{2}=k_{i}\left|\tilde{\mu}_{i}\right|^{2}=\tilde{k}_{i}$ for given $k_{i}$ and $\tilde{k}_{i}$. We point out that already here we introduced complex amplitudes. These are amplitudes of velocities of fluctuations (in Alice's brain) between various possibilities. Thus, although the system of equations (5) looks as purely classical dynamics, the quantum-like structure of decision ${ }^{433}$ making is already incorporated in it, through these com- ${ }_{434}$ plex amplitudes. The system of equations (5) depends 435 on the prediction state, i.e., the state of possible Bob's ${ }_{436}$ actions which was created in Alice's brain. This is an ${ }^{437}$ example of so called adaptive dynamics [28]. In prin- ${ }_{438}$ ciple, adaptive dynamics in combination with complex 439 amplitudes of velocities is sufficient to reproduce sta- 440 tistical data obtained in experiments on the disjunction 441 effect [24], [25], [26]. As was mentioned, this dynamics 442 can be represented as a quantum-like dynamics of den- ${ }^{443}$ sity operator, (12). The use of the quantum-like form ${ }_{444}$ for dynamical equations simplifies essentially the formalism and provides a possibility to use standard mathematical methods of quantum mechanics and quantum information theory. However, this is not the main reason to apply the quantum-like representation of dynamical equations. The main reason is that we proceed under the basic assumption that the brain (as a macroscopic neuronal system) really uses the vector representation of probabilities.

\subsection{Quantum-like Model of Irrational Behavior}

The parameters $\left(k_{i}, \tilde{k}_{i}\right)$ introduced in the previous subsection specify the player's four kinds of comparisons, see Eq. (7). It is natural that these comparisons depend on a given game, namely its pay-off table like $(a, b, c$

\begin{tabular}{|c|c|c|}
\hline$A \backslash B$ & $0_{B}$ & $1_{B}$ \\
\hline $0_{A}$ & $(a \backslash a)$ & $(b \backslash c)$ \\
\hline $1_{A}$ & $(c \backslash b)$ & $(d \backslash d)$ \\
\hline
\end{tabular}

and $d$ in the above table mean values of pay-offs.) The most simple relation of pay-offs and parameters $\left(k_{i}, \tilde{k}_{i}\right)$ can be obtained via depending on magnitude relation between values of pay-off. In the case of PD-type game, the relation of pay-offs is $c>a>d>b$, and then, $k_{i}$ and $\tilde{k}_{i}$ are given as

$$
\begin{aligned}
& k_{1}=1, k_{2}=1, k_{3}=1, k_{4}=0, \\
& \tilde{k}_{1}=0, \tilde{k}_{2}=0, \tilde{k}_{3}=0, \tilde{k}_{4}=1 .
\end{aligned}
$$

Such setting is simple, but not real. The real player's decision-making will depend on differences between pay-offs, not only magnitude relations. That is, the following setting will be more realistic

$$
\begin{gathered}
k_{1}=f_{1}(|a-c|), k_{2}=f_{2}(|b-d|), k_{3}=f_{3}(|b-c|), k_{4}=0 ; \\
\tilde{k}_{1}=0, \tilde{k}_{2}=0, \tilde{k}_{3}=0, \tilde{k}_{4}=\tilde{f}_{4}(|a-d|) .
\end{gathered}
$$

The functions $f_{i}(x)$ are assumed to be monotone increasing functions.

Under the settings of $k_{i}$ and $\tilde{k}_{i}$ of (9) or (10), the probability $P_{0 A}^{E}$ of Eq. (6) is non-zero as a result. Thus, our model explains that the player A generally has potential to make the "irrational" choice of 0 in the PD-game. The reason for this result is that the parameter of $\tilde{k}_{4}$ is non-zero. The $\tilde{k}_{4}$ represents the degree of tendency to choose 0 which occurs from the comparison between consequences of $0_{A} 0_{B}$ and $1_{A} 1_{B}$. It should be noted that such comparison is not considered in classical game theory. 


\section{Dynamics of Alice's Mental State, Density Matrix ${ }_{463}$}

In our model, the dynamics of probabilities corresponding to the mental state is specified by the differential equations Eq. (5):

$$
\begin{aligned}
& \frac{d}{d t} P_{0_{A}}=-k P_{0_{A}}+\tilde{k} P_{1_{A}}, \\
& \frac{d}{d t} P_{1_{A}}=k P_{0_{A}}-\tilde{k} P_{1_{A}} .
\end{aligned}
$$

Here, $P_{0_{A}}=|x|^{2}$ and $P_{1_{A}}=|y|^{2} . \quad(x$ and $y \in \mathbf{C} 470$ are coefficients of the mental state $|\Psi\rangle=x\left|0_{A}\right\rangle \otimes$ $\left|\phi_{B}\right\rangle+y\left|1_{A}\right\rangle \otimes\left|\phi_{B}\right\rangle=x\left|\Phi_{0_{A}}\right\rangle+y\left|\Phi_{1_{A}}\right\rangle$, where $\left|\phi_{B}\right\rangle=$ $\alpha\left|0_{B}\right\rangle+\beta\left|1_{B}\right\rangle \alpha, \beta \in \mathbf{C}$ is the prediction vector defined in Eq. (1). The parameters $k$ and $\tilde{k}$ are defined in (8).

In this section, we complete the dynamics Eq. (5) to a quantum dynamics of the density matrix $\rho=|\psi\rangle\langle\psi|$,

$$
\rho=\left(\begin{array}{cc}
\rho_{00} & \rho_{01} \\
\rho_{10} & \rho_{11}
\end{array}\right) .
$$

corresponding to the mental state $|\psi\rangle=x\left|0_{A}\right\rangle+y\left|1_{A}\right\rangle$ describing the superposition of A's decisions. Details of derivation (based on discrete quantum Markov chains) can be found in [14]. Here we present the final dynamical equation:

$$
\begin{aligned}
& \frac{d}{d t} \rho_{00}(t)=-\left|\mu_{0}\right|^{2} \rho_{00}+\left|\mu_{1}\right|^{2} \rho_{11}, \\
& \frac{d}{d t} \rho_{01}(t)=-\frac{1}{2} \rho_{01}+\mu_{0}^{*} \mu_{1} \rho_{10}, \\
& \frac{d}{d t} \rho_{10}(t)=-\frac{1}{2} \rho_{10}+\mu_{0} \mu_{1}^{*} \rho_{01}, \\
& \frac{d}{d t} \rho_{11}(t)=\left|\mu_{0}\right|^{2} \rho_{00}-\left|\mu_{1}\right|^{2} \rho_{11},
\end{aligned}
$$

where

$$
\left|\mu_{0}\right|^{2}=\frac{|\mu|^{2}}{|\mu|^{2}+|\tilde{\mu}|^{2}},\left|\mu_{1}\right|^{2}=\frac{|\tilde{\mu}|^{2}}{|\mu|^{2}+|\tilde{\mu}|^{2}} .
$$

It is clear that the equations for diagonal parts correspond to the equations (5) essentially. ${ }^{7}$

\footnotetext{
${ }^{7}$ We state again that the dynamics (12) is an oversimplified version of the GKSL-equation. In general, the equations for the diagonal terms contain the off-diagonal terms and vice versa. Surprisingly enough, already the equation (12) describes the process of decision making which is essentially different from the classical Markovian decision making. We shall see that the presence of the off-diagonal terms decreases the entropy of the mental state. In some way offdiagonal terms monitor the interaction with memory, the mental bath, in the process of decision making. In the quantum-like regime the brain uses less memory in the process of decision making, i.e., it performs a more optimal selection of information for decision making.
}

In this dynamics, any initial state of $\rho(0)$ approaches the unique equilibrium state $\rho_{E}$;

$$
\rho_{E}=\left(\begin{array}{cc}
\left|\mu_{0}\right|^{2} & 0 \\
0 & \left|\mu_{1}\right|^{2}
\end{array}\right),
$$
po

\section{1 \\ 4. Dynamics of Entropy in the Process of Decision Making}

As usual in quantum information theory, we can consider von Neumann entropy, see, e.g., [27], of the quantum (mixed) state $\rho(t)$ and study its dynamics, $S(t)=$ $-\operatorname{Tr} \rho(\mathrm{t}) \ln \rho(\mathrm{t})$. This dynamics depends nontrivially on the initial state $\rho\left(t_{0}\right)$ as well as amplitudes of velocities of fluctuations between various possibilities in Alice's brain. In our model Alice's initial state of mind is always pure, this is

$$
\left|\phi_{A}\right\rangle=x_{0}\left|0_{A}\right\rangle+y_{0}\left|1_{A}\right\rangle,
$$

$\left|x_{0}\right|^{2}+\left|y_{0}\right|^{2}=1$. The state $\left|\Psi_{A}\right\rangle$, see (4), can be written as

$$
\left|\Psi_{A}\right\rangle=\left|\phi_{A}\right\rangle \otimes\left|\phi_{B}\right\rangle .
$$

473 (We state again that the predictions state $\left|\phi_{B}\right\rangle$ providpossible Bob's actions has been already used to determine dynamics of $\rho(t)$, see (8).) The density matrix $\rho\left(t_{0}\right)$ corresponding to the pure state $\left|\phi_{A}\right\rangle$ has the form

$$
\rho\left(t_{0}\right)=\left(\begin{array}{ll}
\left|x_{0}\right|^{2} & x_{0} y_{0}^{*} \\
x_{0}^{*} y_{0} & \left|y_{0}\right|^{2}
\end{array}\right),
$$

We can always assume that $x_{0}$ is a real number, $0 \leq$ $x_{0} \leq 1$, and

$$
y_{0}=\sqrt{1-x_{0}^{2}} e^{i \theta_{A 0}} .
$$

We shall not play with all parameters involved in complex amplitudes $\mu_{0}$ and $\mu_{1}$. We just select $\mu_{0}$ as a real parameter and

$$
\mu_{1}=\sqrt{1-\mu_{0}} e^{i \theta_{\mu}} .
$$

Figure 1 contains graphs of quantum entropy $S_{1}(t), S_{2}(t), S_{3}(t)$ corresponding to three different choices of parameters:

$S_{1}$, solid line : $\mathrm{x}_{0}=0.33, \mu_{0}=0.85, \theta_{\mathrm{A} 0}=0.1, \theta_{\mu}=0.2$; 
$S_{2}$, dashed : $\mathrm{x}_{0}=0.315, \mu_{0}=0.625, \theta_{\mathrm{A} 0}=0.1, \theta_{\mu}=0.2 ; 513$ $S_{3}$, dotted : $\mathrm{x}_{0}=0.99, \mu_{0}=0.925, \theta_{\mathrm{A} 0}=0.1, \theta_{\mu}=0.2$.

(So, phases are the same for all graphs, we play only with amplitudes.) These graphs show that behavior of quantum entropy depends essentially on parameters, i.e., the initial state of Alice's mind and velocities of fluctuations between various possibilities.

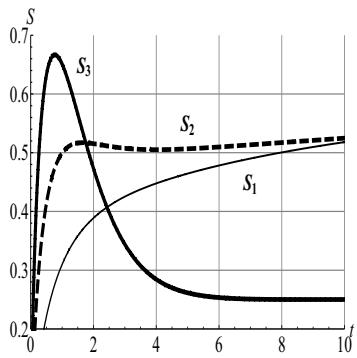

Figure 1: Dynamics of quantum entropy

There are two main forms of graphs:

1). Entropy monotonically increases by stabilizing to a constant value; the first stage of the process of decision making is characterized by very quick increasing of entropy, then entropy increases very slowly; for large $t$, it varies negligibly, see the graph of $S_{1}$.

We see that the first period of decision making is characterized by attraction of a large amount of new information (mainly from memory, but from the external environment as well); entropy increases very quickly. Then, when the processing of this information is more or less finished, entropy stabilizes (becoming a constant) by approaching the equilibrium point.

2). Entropy increases very quickly by approaching a local maximum, then it decreases and, finally, stabilizes. Typically, on the way to stabilization it has a local minimum and then it increases again.

In both cases, at the initial stage of decision making entropy increases as the result of interaction with "environment" (in particular, memory), then (for some clusters of parameters) it decreases and uncertainty is mini- ${ }_{525}$ mized, see $S_{2}, S_{3}$, but then (before the final step of de- ${ }_{526}$ cision making) it increases again. Sometimes this final ${ }_{527}$ increasing of entropy is negligibly small, see the graph ${ }_{528}$ of $S_{3}$; sometimes entropy increases visibly, see $S_{2}$. ${ }_{529}$

The dynamics of quantum entropy represents the dy- ${ }_{530}$ namics of uncertainty in Alice's decision state, entropy ${ }_{531}$ of choices between strategies. However, it does not de- 532 scribe the dynamics of Alice's diffidence to make the ${ }_{533}$ decision at this point $t$. We are looking for a quantity ${ }_{534}$

which can be considered as a measure of Alice's diffidence.

First of all we introduce classical entropy corresponding to the diagonal terms in the density matrix $\rho(t)$. This is entropy for Alice's choice between two possible decisions if she were ignoring the off-diagonal terms. The presence of these terms is a consequence the quantumlike representation of information in the brain. The complete mental state of Alice is a pure state (of a huge dimension); the density matrix of Alice's decision state is extracted from this state. The quantum-like dynamics of this density matrix induces not only dynamics of the diagonal terms, but even off-diagonal ones. We set

$$
S C L(t)=-\left(\rho_{00}(t) \ln \rho_{00}(t)+\rho_{11}(t) \ln \rho_{11}(t)\right),
$$




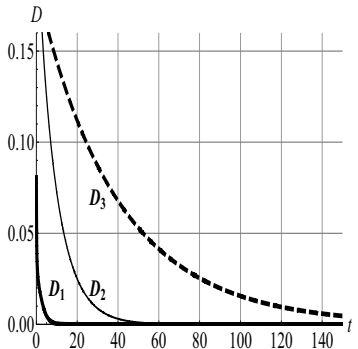

Figure 3: Dynamics of diffidence

but it can fluctuate depending on context. It is natural ${ }_{582}$ to assume that $\epsilon_{D F}$ has the Gaussian distribution. In this model decision times are random, since the difference threshold is random. ${ }^{8}$

\section{On experimental verification of dynamics of men- tal quantum-like state; coupling to the brain ar- chitecture}

One of the referees of this paper asked a important 591 question:

"How to measure or observe the dynamics of quan- ${ }^{593}$ tum entropy and difference during decision making in 594 $\mathrm{PD}$, in neurobiological, behavioral or psychological ex- ${ }^{595}$ periments?"

In principle, the formalism of quantum information ${ }^{59}$ theory provides a possibility to reconstruct a density matrix on the basis of statistical data obtained through 599 a series of measurements of a family of incompatible 600 observables - quantum tomography. Since observations ${ }^{601}$ are incompatible, we are not able to perform them on ${ }^{602}$ the same biological system: human person, animal, nor ${ }^{603}$ even insect. Therefore the state reconstruction (at each ${ }^{604}$ instance of time) will demand a large statistical ensem- ${ }^{605}$ ble of biological systems participating in experiments. ${ }^{600}$ In theory the family of incompatible observables depend ${ }^{607}$ on continuous parameters; in reality we discretize these ${ }^{608}$ parameters. However, to obtain a good approximation, the discretization has to be sufficiently fine. Thus even for one instance of time the experiment demands a huge group of participants. Moreover, members of such a group should behave homogeneously. It is not clear whether it would be possible to perform experiments on monitoring of mental state dynamics in PD experiments with people. We speculate that it may be possible to design PD-type games for simpler biological systems:

\footnotetext{
${ }^{8} \mathrm{~A}$ similar problem was discussed in [12].
}

animals? may be even insects? In the later case we have a better chance to create samples which behave homogeneously. This is an interesting problem addressed to psychologists and cognitive scientists.

However, it is too early to concentrate studies on the aforementioned problem. The following fundamental problem is essentially more important. In conventional quantum tomography we have to be able to measure linear combinations of incompatible observables. For example, let $\hat{p}$ and $\hat{q}$ denote the momentum and position operators, respectively. We also have to measure rotations of the type $\hat{z}_{\theta}=\cos \theta \hat{q}+\sin \theta \hat{p}, \theta \in[0,2 \pi)$. In quantum physics such measurement can be reduced to rotation of the state. And nowadays the technology of quantum state manipulation is well developed, so there is no problem to perform a state rotation. ${ }^{9}$ We have to develop a similar technology of mental state manipulation in biology. At the moment it is too early to speculate about a possibility of realization of this exciting project. In biology the situation is more complicated than in physics: there is no (or at least it is not yet known) coupling between the structure of space and mental states. For example the polarization vector is still meaningful in quantum physics ${ }^{10}$. Therefore we can use the spacerepresentation of classical physics to prepare quantum systems in special states of polarization.

Another important question is about the neuronal architecture of quantum-like dynamics of decision making. By our model the dynamics described by the quantum master equation is realized on neuronal level. It describes interaction of the mental state encoding possible decisions with mental environment. As was remarked in [16]: "An optimal action, namely an action that is most likely to yield the most desirable outcome, often changes according to the state of the animals environment. Therefore, if a sensory stimulus informs the animal of a change in the state of its environment, the animal needs to store such information until it produces an appropriate action or until this information can be combined with another stimulus to determine the new state of the environment." In that paper it was pointed to the lat-

\footnotetext{
${ }^{9}$ We point to the tremendous development of quantum technologies of state manipulation during the last ten years. This development was stimulated by large interest to quantum computing and cryptography. At the very beginning of quantum mechanics there was a huge gap between theoretical consideration involving all possible quantum states and possibilities for experimental realization of these states.

${ }^{10}$ The situation is more delicate. In quantum physics the polarization cannot be defined in the same way as in classical electrodynamics. If the polarization vector were well defined, it would serve as a hidden variable. As a consequence of Bell's theorem, the latter does not exist. (This viewpoint has not been commonly accepted, see e.g. [11]). In any event the operator of projection of polarization to any direction is well defined.
} 
eral prefrontal cortex as responsible for the state repre- 651 sentation of the mental environment. We may speculate ${ }_{652}$ that the linear operator of the quantum master equation, 653 see appendix, is stored in this domain of brain. (In the 654 real situation this operator is time dependent.)

In [15], [16] it was also remarked that lesions which 656 encompass the orbitofrontal cortex in human patients as 657 well as psychiatric conditions as substance abuse and 658 frontotemporal dementia may crucially change the abil- 659 ity to represent the statistical interdependence between 660 the values of alternative options [16]. Knowledge of 661 such interdependence might allow the decision makers 662 to switch to a better choice immediately, as soon as 663 they experience the reduction of values from a partic- 664 ular choice. We may speculate that the quantum-like 665 representation of the mental state (by a density matrix) 666 is performed in the orbitofrontal cortex. The density ${ }_{667}$ matrix describes correlations between alternative op- 668 tions; it seems that lesions in the orbitofrontal cortex 669 and aforementioned psychiatric conditions destroy (at ${ }_{670}$ least partially) the density matrix.

Conclusive remarks. We elaborated a quantum-like model of decision making based on interaction between the decision-state, i.e., the state carrying probabilities for possible choices, and the state of memory (or more general mental environment). We can speculate that decoherence may be a key element of transition from quantum unconsciousness (in which mind is represented by vector superpositions) to classical consciousness (in which the mind is represented by classical probabilistic mixtures). ${ }^{11}$

\section{Appendix: Mathematical formalism of quantum mechanics}

The mathematical formalism of quantum mechanics describes states of systems and observables, see book ${ }^{682}$ [11] for a simple representation of quantum mathemat- ${ }^{683}$ ics for biologists and psychologists. (The quantum- ${ }^{684}$ like approach is based on the observation that "quantum ${ }^{685}$ mathematics" can be applied outside quantum physics.) ${ }^{686}$

The basic mathematical structure of quantum me- ${ }^{687}$ chanics is a complex Hilbert space: a linear space over 688 complex numbers $H$ (i.e., it is possible to form linear 689

\footnotetext{
${ }^{11}$ Thus, mind is always probabilistic, but it has different representations in consciousness and unconsciousness. The process of interaction of the decision-state with memory can be described by the same model as decoherence of a pure state of a quantum system interacting with an environment, a bath; memory is a kind of mental bath for decision states.
}

combinations of vectors with complex coefficients) endowed with a Hermitian bilinear form mapping a pair of vectors $\psi_{1}, \psi_{2} \in H$ into a complex number denoted $\left\langle\psi_{1} \mid \psi_{2}\right\rangle$ (we use Dirac's notation which is typical for quantum information theory). We shall be interested in complex vectors normalized by one, i.e., $\psi \in H$ such that $\|\psi\|^{2}=\langle\psi \mid \psi\rangle=1$. Such vectors encode so called pure states of quantum systems. Normalization by one is crucial for the probabilistic interpretation of pure states. Observables (e.g., the energy-observable or the position observable) are encoded by self-adjoint operators.

The theory is especially simple in the finite dimensional case (which is typically considered in quantum information theory). Here $H=\mathbf{C}^{n}$ is the Cartesian product of $n$-copies of the set of complex numbers $\mathbf{C}$. Hence, a pure state $\phi=\left(z_{1}, \ldots, z_{n}\right)$, where $\|\psi\|^{2}=$ $\left|z_{1}\right|^{2}+\ldots+\left|z_{n}\right|^{2}=1$. Observables are given (in an orthonormal basis) by Hermitian matrices $A=\left(a_{i j}\right)$; here $\bar{a}_{i j}=a_{j i}$. In the Dirac notation the matrix elements are written as $\left\langle\phi_{1}|A| \phi_{2}\right\rangle$. Coordinates of a state vector are interpreted as probabilities (Born's interpretation of a pure quantum states [11]). One can say that the quantum formalism provides a geometric representation of probabilities which is based on the Euclidean distance.

Dynamics of a pure state is described by the Schrödinger equation

$$
i \frac{d \psi}{d t}(t)=\mathcal{H} \psi(t), \psi(0)=\psi_{0},
$$

where the operator $\mathcal{H}$ is the generator of evolution (Hamiltonian, the operator of energy). We remark that Schrödinger dynamics is deterministic. By fixing the initial state we can find the state of a quantum system at any instance of time. The fundamental problem of quantum foundations is that this state does not determine values of observables. Measurements induce randomness.

We remark that each pure state $\psi$ determines a selfadjoint operator, the projector onto this state; $\rho \equiv|\psi\rangle\langle\psi|$ (the last symbol is simply the Dirac notation): $\rho \phi=$ $\langle\phi \mid \psi\rangle \psi$. We recall the basic properties of $\rho_{\psi}$ :

a) it is positively defined, i.e., $\langle\phi|\rho| \phi\rangle \geq 0$ for any $\phi$;

b) it is Hermitian;

c) its trace (the sum of diagonal elements) equals to one.

The Schrödinger dynamics for pure states (vectors) can be rewritten as dynamics for corresponding operators:

$$
i \frac{d \rho}{d t}(t)=[\mathcal{H}, \rho(t)], \rho(0)=\rho_{0},
$$


where $[\mathcal{H}, \rho]=\mathcal{H} \rho-\rho \mathcal{H}$ is the commutator of opera- 722 tors.

Consider now a statistical mixture (in the classical sense) of a few projection operators $\rho_{i}$ corresponding ${ }_{724}^{723}$ to pure states $\psi_{i}$ with weights $p_{i} \geq 0, \sum p_{i}=1$,

$$
\rho=p_{1} \rho_{1}+\ldots+p_{n} \rho_{n} .
$$

Each operator of this form satisfies conditions a) $-\mathrm{c}$ ) and vice versa. Denote the class of all operators with properties a)-c) by the symbol $\mathcal{D}(H)$. This is the space of ${ }_{731}$ states of quantum systems. Its elements (called density ${ }^{732}$ operators) can be interpreted as statistical mixtures of ${ }^{733}$ pure states. In general a density operator can be rep- ${ }_{735}^{734}$ resented in the form (19) in many ways. There is one 736 special expansion corresponding to eigenvectors of $\rho$. ${ }^{737}$ The density operator corresponding to a pure state can ${ }^{738}$ be characterized in the following way: in the basis of ${ }_{740}^{739}$ eigenvectors, its matrix has only one nonzero element ${ }^{741}$ (equal to one), i.e., up to a permutation of eigenvectors:

$$
\rho=\left(\begin{array}{ll}
1 & 0 \\
0 & 0
\end{array}\right)
$$

where the blocks of zeros have the corresponding sizes. However, this takes place only in the basis of eigenvec- ${ }^{748}$ tors, cf. (16).

Dynamics of a quantum state is described by the ${ }^{750}$ equation (18), von Neumann equation. This dynamical 752 model can be used only in the absence of interaction of 753 a quantum system with an environment, a bath. If such interaction is essential (so a system cannot be considered as isolated), von Neumann dynamics (18) has to be modified and additional terms have to be included in it. The basic postulate of quantum theory is that the state dynamics is linear. Therefore modified dynamics has the form

$$
i \frac{d \rho}{d t}(t)=[\mathcal{H}, \rho(t)]+L(\rho), \rho(0)=\rho_{0},
$$

where $L$ is a linear operator. This operator has to be chosen in such a way that starting with $\rho_{0} \in \mathcal{D}(H)$, we shall obtain a trajectory $t \rightarrow \rho(t)$ in $\mathcal{D}(H)$. The corre- 76 sponding conditions on linear systems were formulated by Gorini, Kossakowski, Sudarshan, and Lindblad, see, e.g., [27] for details.

\section{acknowledgments}

Research of one of the authors (Irina Baseiva) is supported by the post-doc fellowship of Swedish Institute; 70 this paper was completed during the visit of two authors 78 (Irina Baseiva and Andrei Khrennikov) to the QBIC Center of Tokyo University of Science (March and October 2010).

\section{References}

[1] A. Khrennikov, Quantum-like brain: Interference of minds, BioSystems 84 (2006) 225-241.

[2] J. R. Busemeyer, Z. Wang, and J. T. Townsend, Quantum dynamics of human decision making, J. Math. Psychol. 50 (2006) 220-241.

[3] P. La Mura: Projective expected utility, in: Bruza, P. D., Lawless, W., van Rijsbergen, K., Sofge, D. A., Coecke, B. and Clark, S. (Eds.), Quantum Interaction-2, College Publications, London, 2008, pp. 87-93.

[4] K.-H. Fichtner, L. Fichtner, W. Freudenberg, and M. Ohya, On a quantum model of the recognition process, QP-PQ: Quantum Prob. White Noise Analysis 21 (2008) 64-84.

[5] L. Accardi, A. Khrennikov, M. Ohya, The problem of quantumlike representation in economy, cognitive science, and genetics, in: L. Accardi, W. Freudenberg, M. Ohya (Eds.), Quantum BioInformatics II: From Quantum Information to Bio-Informatics, WSP, Singapore, 2008, pp. 1-8.

[6] L. Accardi, A. Khrennikov, M. Ohya, Quantum Markov model for data from Shafir-Tversky experiments in cognitive psychology, Open Systems and Information Dynamics 16 (2009) 371385.

[7] E. Conte, A. Khrennikov, O. Todarello, A. Federici, J. P. Zbilut, Mental states follow quantum mechanics during perception and cognition of ambiguous figures, Open Systems and Information Dynamics 16 (2009) 1-17.

[8] A. Khrennikov and E. Haven, Quantum mechanics and violations of the sure-thing principle: the use of probability interference and other concepts, J. Math. Psychology 53 (2009) 378388.

[9] A. Khrennikov, Quantum-like model of cognitive decision making and information processing, Biosystems 95 (2009) 179-187.

[10] R. Franco, The conjunction fallacy and interference effects, J. Math. Psychol. 53 (2009 415-422.

[11] A. Khrennikov, Ubiquitous Quantum Structure: from Psychology to Finance, Springer, Heidelberg-Berlin-New York, 2010.

[12] E. M. Pothos, J. R. Busemeyer, A quantum probability explanation for violation of rational decision theory, Proc. Royal. Soc. B, 276 (2009) 2171-2178.

[13] T. Cheon and T. Takahashi, Interference and inequality in quantum decision theory, Phys. Lett. A 375 (2010) 100-104.

[14] M. Asano, A. Khrennikov, M. Ohya, Quantum-like model for decision making process in two players game. A NonKolmogorovian model, Found. Phys. 41, 538-548 (2010).

[15] D. J. Barraclough, M. L. Conroy and D. Lee, Prefrontal cortex and decision making in a mixed-strategy game, Nature Neuroscience 7 (2004) 404 - 410.

[16] D. Lee, M. F. S. Rushworth, M. E. Walton, M. Watanabe, and M. Sakagami, Functional specialization of the primate frontal cortex during decision making, J. Neurosci. 27 ( 2007) 81708173.

[17] T. Inada, K. Kimata, H. Aiba, Mechanism responsible for glucose-lactose Diauxie in Escherichia Coli challenge to the cAMP model, Genes and Cells 1 (1996) 293-301.

[18] K. Nakao, R. Morita, Y. Saji, K. Ishida, Y. Tomita, M. Ogawa, M. Saitoh, Y. Tomooka, and T. Tsuji, The development of a bioengineered organ germ method, Nature methods 4 (2007) 227230.

[19] S. Hameroff, Quantum coherence in microtubules. A neural basis for emergent consciousness? J. Consciousness Stud. 1 (1994) 91-118.

[20] S. Hameroff, Quantum computing in brain microtubules? The Penrose-Hameroff "Orch or model of consciousness, Philos. Trans. R. Soc. London A 356 (1998) 1869-1896. 
[21] R. Penrose, The Emperor's New Mind, Oxford Univ. Press, New-York, 1989.

[22] R. Penrose, Shadows of the Mind, Oxford University Press, Oxford, 1994.

[23] A. Khrennikov, On the physical basis of theory of "mental waves", Neuroquantology,

[24] E. Shafir and A. Tversky, Thinking through uncertainty: nonconsequential reasoning and choice, Cogn. Psychol. 24 (1992) 449-474.

[25] A. Tversky and E. Shafir, The disjunction effect in choice under uncertainty, Psychol. Sc. 3 305-309 (1992)

[26] R. Croson, The disjunction effect and reasoning-based choice in games, Organizational Behavior and Human Decision Processes 80 (1999) 118-133.

[27] R. S. Ingarden, A. Kossakowski, and M. Ohya, Information Dynamics and Open Systems: Classical and Quantum Approach, Kluwer, Dordrecht, 1997.

[28] M. Ohya, Adaptive dynamics and its applications to chaos and NPC problem, QP-PQ: Quantum Prob. White Noise Anal. 21 (2008) 186-216.

[29] L.J. Savage, The Foundations of Statistics, Wiley and Sons, New York, 1954.

[30] J. Von Neumann and O. Morgenstern, Theory of Games and Economic Behavior, Princeton University Press, Princeton, 1947.

[31] I. Gilboa, A. Postlewaite and D. Schmeidler, Is it always rational to satisfy Savage axioms? Economics and Philosophy 25 (2009) 285-296.

[32] J. Eisert, M. Wilkens, and M. Lewenstein, Quantum games and quantum strategies, Phys. Rev. Lett. 83 (1999) 3077-3081. G.R. Mettam, L.B. Adams, How to prepare an electronic version of your article, in: B.S. Jones, R.Z. Smith (Eds.), Introduction to the Electronic Age, E-Publishing Inc., New York, 1999, pp. 281304.

[33] R. Cleve, P. Hyer, B. Toner and J. Watrous, Consequences and limits of nonlocal strategies, Proc. 19th IEEE Conference on Computational Complexity (CCC 2004), 2004, pp. 236-249.

[34] T. Cheon and I. Tsutsui, Classical and quantum contents of solvable game theory on Hilbert space, Phys. Lett. A 348 (2006) $147-152$. 
>A novel model of quantum-like processing of mental information is presented.

$>$ This model matches the neuronal paradigm of cognitive science.

>Classical electromagnetic signals provide the basis for quantum-like representation.

>Signals are induced by joint activity of neurons. 